\title{
Progression of lumbar disc degeneration over a decade: a heritability study
}

\author{
Frances M K Williams, ${ }^{1}$ Maria Popham, ${ }^{1}$ Philip N Sambrook, ${ }^{2}$ Annette F Jones, ${ }^{1}$ \\ Tim D Spector, ${ }^{1}$ Alex J MacGregor ${ }^{3}$
}

${ }^{1}$ Department of Twin Research and Genetic Epidemiology, King's College London, London, UK

${ }^{2}$ Kolling Institute, University of Sydney, Sydney, New South Wales, Australia

${ }^{3}$ School of Medicine, University of East Anglia, Norwich, UK

\section{Correspondence to} Dr Frances M K Williams, Department of Twin Research and Genetic Epidemiology, King's College London, St Thomas Hospital, Westminster Bridge Road, London SE1 7EH, UK; frances.williams@kcl.ac.uk

Accepted 5 February 2011 Published Online First 13 March 2011

\section{UNLOCKE}

This paper is freely available online under the BMJ Journals unlocked scheme, see http:// ard.bmj.com/info/unlocked.dtl

\section{ABSTRACT}

Objectives Lumbar disc degeneration (LDD) is prevalent, age-related and contributes to low back pain. Crosssectional LDD as determined by MRI scan is known to be highly heritable. The authors postulated that the rate of progression might also be controlled by genetic factors. Methods A 10-year follow-up of MRI-determined LDD was performed in 234 pairs of twin volunteers in the UK and Australia, comprising 90 monozygotic pairs and 144 dizygotic same-sex twin pairs. Of the total sample, $95 \%$ were female. The mean age at baseline was 53.3 years (range 32.3-69.5). The rate of progression was calculated and, because the effect of age was non-linear, the sample was divided into age strata and heritability estimated for each trait's progression.

Results All MRI-determined traits worsened significantly over the period of follow-up ( $p<0.0001$ for each). Change in disc height was not heritable at any age while posterior disc bulge was heritable across all age categories (range $28-53 \%$ ), with higher heritability in those over 60 years. Change in disc signal intensity and anterior osteophytes were found to be heritable only in those aged under 50 years at baseline (heritability estimates $76 \%(95 \% \mathrm{Cl} 44 \%$ to $100 \%)$ and $74 \%(42 \%$ to $100 \%)$, respectively).

Conclusions Longitudinal change in LDD traits is heritable for all traits except disc height, but there is a significant influence of age, which varies across traits. Future studies to define the genetic variants influencing LDD progression should examine MRI traits individually and in women should focus on those under 50 years of age.

Low back pain is highly prevalent in the western world and accounts for considerable work absenteeism. We and others have shown a relationship between back pain and lumbar disc degeneration (LDD), although the strength of association remains debated. ${ }^{1-4}$ Several twin studies have shown LDD to be highly heritable but to date genetic analysis has focused on cross-sectional data. Previous longitudinal analyses had suggested that progression may be more clinically relevant: the development of radiographic disc space narrowing over 9 years of follow-up, for example, was predicted by the presence at baseline of low back pain in the Chingford study. ${ }^{5}$ In this study, we examined LDD progression longitudinally in monozygotic and dizygotic twins using lumbar spine MRI at two time points, over 10 years apart. We also estimated the heritability of LDD progression.

\section{SUBJECTS AND METHODS}

The study sample comprised same-sex twin pairs recruited from the TwinsUK register and the Sydney Twin Study. Between 1995 and 2001, 981 male and female twins were subjects in the baseline MRI study. ${ }^{6}$ None of the twins was aware of the hypothesis under investigation. The twins were neither selected nor excluded if there was a history of lower back pain, neck pain or degenerate disc disease. This sample represents part of an adult female twin cohort recruited since 1995 (www.twinsuk.ac.uk) from the general UK population $^{7}$ and from the Australian Twin Registry. ${ }^{8}$ The twins attended for MRI scan and a visit with a nurse metrologist to collect demographic data. Appropriate ethics permission had been obtained by both groups and twins gave informed written consent at both time points of the study.

\section{MRI at baseline}

The baseline MRI was performed using a Siemans 1.0T superconducting magnet (Siemans, Munich, Germany) in London and a GE Signa (GE, London, UK) 1.5T in Sydney. Sagittal images were obtained using a fast spin-echo sequence of repetition time/ echo time 5000-4500/112 ms with a slice thickness of $4 \mathrm{~mm}{ }^{6}$ Serial sagittal images of the thoracolumbar junction and lumbar spine (T9-L5) were obtained as described in detail, including reliability scores, elsewhere. At each disc level the main traits were scored by PNS considering the middle slices of the scan and using a progressive scale of $0-3$ for: disc height measured in the middle of the disc; disc signal intensity within the nucleus pulposus; lumbar disc extension posteriorly into the spinal canal and anterior osteophytes. A score of 0 indicates a normal disc, whereas a score of 3 is given for a highly degenerate disc. A summary score for the different traits was produced, by summing the coding grades for the region L1/2 to L5/S1. Twins of a pair were scanned on the same day, in the morning, more than $1 \mathrm{~h}$ after rising.

\section{MRI at follow-up}

The twins included in the baseline study were recontacted between 2007 and 2009 and approximately half the pairs re-attended for a clinical visit and further spine MRI scans. Scan parameters reproduced those used at baseline (Figure 1). All imaging departments we contacted in London had upgraded their machines at some point over the intervening decade. Repeat scanning was performed on a Siemans 1.5T in London and a different Signa 1.5T in Sydney with the same slice measurements and excitation time and relaxation time as previously. Serial sagittal images of the thoracolumbar junction and lumbar spine (T9-L5) were obtained and coded for degenerative change using the atlas of Jarosz 
et al, ${ }^{9}$ as previously described in the baseline spine MRI study. ${ }^{6}$ MP was trained in coding by PNS who had coded the baseline scans. In brief, at each disc level the five middle slices of the sagittal MRI were used to score, on a 0-3 scale: disc height measured in the middle of the disc; disc signal intensity within the nucleus pulposus; lumbar disc extension into the spinal canal and anterior osteophytes. A score of 0 indicates a normal disc and a score of 3 a highly degenerate disc. Scans were coded blindly, without reference to baseline coding, scan or to co-twin's results. For each trait, the baseline score was subtracted from the follow-up score to give a change in score. Because the interval between the two scans varied among the twins, the score was adjusted for the time interval between the two MRI scans-effectively giving a rate of change per year. Reproducibility scores were performed intracoder (mean of the four traits 0.68 ; comprising disc height 0.77 , disc signal 0.61 , disc bulge 0.67 , anterior osteophytes 0.66 ) and intercoder (mean 0.54; disc height 0.66, disc signal 0.62, disc bulge 0.56 , anterior osteophytes 0.32 ).

\section{Statistical methods}

Comparisons were made using t tests, the Wilcoxon signed rank test and multivariate regression analysis as appropriate using Stata 2007. Intraclass correlations were calculated for monozygotic and dizygotic twins. The underlying distribution of change in score is considered to reflect contributions by additive genetic (A) factors, shared environmental factors (C) and additive environmental factors (E), and the influence of each was modelled using the De Fries Fulker regression approach implemented in Stata. The effect of age on the rate of progression was non-linear in this dataset so the analysis was carried out in three baseline age strata: 50 years or less; $50-60$ years; 60 years or over.

\section{RESULTS}

Longitudinal data were available for 234 same-sex twin pairs of confirmed zygosity, comprising 90 pairs of monozygotic and
144 pairs of dizygotic twins. The majority (211 pairs) were from the TwinsUK cohort, the remaining 23 pairs were recruited in Australia. Twenty-four subjects (5.1\%) were male, distributed evenly between the two zygosities. Table 1 shows the characteristics of the total sample by zygosity. Mean age at baseline was 53.6 years (range 40.1-68.7), with mean age in monozygotic twins 55.4 years and dizygotic twins 52.6 years. The mean interval between baseline and follow-up was 10.7 years (range 7.6-13.7). The Australian twins were significantly older (Australian vs UK 56.2 vs 53.4 years, $\mathrm{p}=0.01$ ) and heavier (body mass index (BMI) 27.6 vs $24.5 \mathrm{~kg} / \mathrm{m}^{2}, \mathrm{p}<0.01$ ) than the UK twin volunteers, but there was no difference in baseline levels of LDD between the two groups. In general, the monozygotic twins had more advanced degenerative change at baseline (table 1), with a significantly greater osteophyte score and disc signal in the monozygotic compared with the dizygotic twins. These differences were attributable to age: when considered in age strata the baseline traits did not differ significantly between the zygosities (table 1). There was no significant difference between twins attending for follow-up and those not attending in terms of age and baseline summary LDD score but there was a difference in one of the MRI subtraits, osteophytes, with those not followed up having significantly greater baseline levels than those re-attending (1.79 vs 1.14, respectively, $\mathrm{p}<0.001)$.

\section{Progression of LDD}

All MRI traits showed an increase in score over the period of follow-up (table 2), indicative of a deterioration in LDD. In fact, almost all individuals demonstrated deterioration over the period of follow-up: only one person $(0.2 \%)$ had the same summary LDD score at both time points and one person $(0.2 \%)$ had a score that had marginally improved. The change in summary score over the period of follow-up was highly significant, the mean LDD summary score at baseline versus follow-up 12.96 versus $22.97(p<0.0001)$. Dizygotic twins were found to have

Table 1 Sample characteristics at baseline by zygosity, showing mean MRI trait scores for whole group and by age strata

\begin{tabular}{|c|c|c|c|c|}
\hline & Age, years & Monozygotic twins & Dizygotic twins & p Value \\
\hline Sample size & & 180 & 288 & \\
\hline Age, years, mean (range) & & $55.42(40.89-68.74)$ & $52.64(40.08-68.75)$ & $<0.01$ \\
\hline Males, $\mathrm{n}(\%)$ & & 12 & 12 & 0.233 \\
\hline Body mass index, kg/m² (range) & & $24.39(18.85-42.31)$ & $25.01(17.51-45.14)$ & 0.10 \\
\hline \multirow[t]{4}{*}{ Lumbar disc height score, mean (range) } & All ages & $4.07(0-13)$ & $3.80(0-12)$ & 0.25 \\
\hline & $<50$ & $3.08(1.59)$ & $3.48(2.24)$ & 0.33 \\
\hline & $50-60$ & $3.95(2.44)$ & $3.80(3.36)$ & 0.64 \\
\hline & $>60$ & $5.2142(3.15)$ & $4.49(2.71)$ & 0.23 \\
\hline \multirow[t]{4}{*}{ Lumbar disc signal score, mean (range) } & All ages & $6.21(0-11)$ & $5.65(0-12)$ & 0.03 \\
\hline & $<50$ & $4.19(2.29)$ & $4.64(2.69)$ & 0.37 \\
\hline & $50-60$ & $6.37(2.44)$ & $5.80(2.60)$ & 0.09 \\
\hline & $>60$ & $7.57(2.20)$ & $7.41(2.15)$ & 0.73 \\
\hline \multirow[t]{4}{*}{ Lumbar disc bulge score, mean (range) } & All ages & $2.15(0-8)$ & $1.97(0-10)$ & 0.24 \\
\hline & $<50$ & $1.83(1.42)$ & $1.87(1.64)$ & 0.89 \\
\hline & $50-60$ & $2.12(1.707)$ & $1.84(1.54)$ & 0.20 \\
\hline & $>60$ & $2.5(1.81)$ & $2.47(1.77)$ & 0.94 \\
\hline \multirow[t]{4}{*}{ Lumbar anterior osteophyte score, mean (range) } & All ages & $1.40(0-7)$ & $0.99(0-10)$ & $<0.01$ \\
\hline & $<50$ & $1.14(1.59)$ & $0.77(1.11)$ & 0.13 \\
\hline & $50-60$ & $1.24(1.16)$ & $0.96(1.22)$ & 0.08 \\
\hline & $>60$ & $2.0(1.82)$ & $1.53(2.06)$ & 0.25 \\
\hline \multirow[t]{4}{*}{ Summary lumbar score, mean (range) } & All ages & $13.84(0-38)$ & $12.41(0-40)$ & 0.02 \\
\hline & $<50$ & $10.25(4.93)$ & $10.77(5.78)$ & 0.63 \\
\hline & $50-60$ & $13.68(6.08)$ & $12.39(6.05)$ & 0.12 \\
\hline & $>60$ & $17.29(6.55)$ & $15.91(6.86)$ & 0.32 \\
\hline
\end{tabular}

Summary lumbar score represente for all four traits over five lumbar disc levels. $\mathrm{p}$ Value is significance value comparing monozygotic with dizygotic twins. 
Table 2 Changes in LDD phenotypes over time, by zygosity

\begin{tabular}{|c|c|c|c|c|c|c|c|c|c|}
\hline \multirow[b]{2}{*}{ Trait } & \multicolumn{3}{|c|}{ Monozygotic twins } & \multicolumn{3}{|c|}{ Dizygotic twins } & \multicolumn{3}{|c|}{ Comparing monozygotic and dizygotic } \\
\hline & $\begin{array}{l}\text { Baseline } \\
\text { score (SD) }\end{array}$ & $\begin{array}{l}\text { Follow-up } \\
\text { score (SD) }\end{array}$ & $\begin{array}{l}\text { Change in } \\
\text { mean score (SD) }\end{array}$ & $\begin{array}{l}\text { Baseline } \\
\text { score }\end{array}$ & $\begin{array}{l}\text { Follow-up } \\
\text { score }\end{array}$ & $\begin{array}{l}\text { Change in } \\
\text { mean score (SD) }\end{array}$ & $\begin{array}{l}\text { p Value } \\
\text { baseline }\end{array}$ & $\begin{array}{l}\text { p Value } \\
\text { follow-up }\end{array}$ & $\begin{array}{l}\text { p Value } \\
\text { change in trait }\end{array}$ \\
\hline Disc height & $4.07(2.58)$ & $6.39(2.65)$ & 2.271 .93 & $3.80(2.44)$ & $6.52(2.82)$ & $2.71(2.14)$ & 0.25 & 0.63 & 0.03 \\
\hline Disc signal & $6.21(2.61)$ & $8.55(2.13)$ & 2.312 .25 & $5.65(2.73)$ & $8.25(1.98)$ & $2.62(2.18)$ & 0.03 & 0.13 & 0.15 \\
\hline Disc bulge & $2.15(1.69)$ & $3.88(2.37)$ & $1.65(1.76)$ & $1.97(1.63)$ & $4.01(2.43)$ & $2.07(2.00)$ & 0.24 & 0.55 & 0.02 \\
\hline Anterior osteophytes & $1.40(1.46)$ & $4.22(2.68)$ & 2.722 .40 & $0.99(1.40)$ & $4.15(2.70)$ & $3.16(2.47)$ & $<0.01$ & 0.76 & 0.06 \\
\hline LDD summary score & $13.84(6.39)$ & $23.05(6.88)$ & 8.954 .27 & $12.41(6.35)$ & $22.93(7.53)$ & $10.55^{*}(4.68)$ & 0.02 & 0.86 & $<0.01$ \\
\hline
\end{tabular}

Mean summary scores for the individual traits over the five lumbar discs and SD are shown for baseline and follow-up scans, with mean change in score over the 10-year period of follow-up, and $\mathrm{p}$ values are shown for comparison between monozygotic and dizygotic twins at baseline, follow-up and for change in trait. Taken together, all traits' changes over 10 years were highly significant, each trait $\mathrm{p}<0.0001$.

LDD, lumbar disc degeneration.

Table 3 Intraclass correlations, modelling and heritability estimates for the MRI traits by age category

\begin{tabular}{|c|c|c|c|c|c|c|c|}
\hline $\begin{array}{l}\text { Age, } \\
\text { years }\end{array}$ & $\Delta$ Trait/time (per year) & $\begin{array}{l}\text { N pairs } \\
\text { (monozygotic) }\end{array}$ & $\begin{array}{l}\text { N pairs } \\
\text { (dizygotic) }\end{array}$ & $\begin{array}{l}\text { R-monozygotic } \\
\text { (95\% CI) }\end{array}$ & $\begin{array}{l}\text { R-dizygotic } \\
\text { (95\% CI) }\end{array}$ & A (95\% Cl) & C (95\% CI) \\
\hline$<50$ & Disc height & 18 & 53 & $0.51(0.16$ to 0.87$)$ & $0.53(0.33$ to 0.72$)$ & & $0.51(0.30$ to 0.71$)$ \\
\hline $50-60$ & Disc height & 50 & 63 & $0.33(0.076$ to 0.57$)$ & $0.40(0.19$ to 0.62$)$ & & $0.37(0.19$ to 0.54$)$ \\
\hline$>60$ & Disc height & 22 & 28 & $0.18(0.00$ to 0.63$)$ & $0.47(0.17$ to 0.77$)$ & & $0.38(0.10$ to 0.65$)$ \\
\hline$<50$ & Disc signal & 18 & 53 & $0.71(0.48$ to 0.95$)$ & $0.41(0.18$ to 0.63$)$ & $0.76(0.44$ to 1.0$)$ & \\
\hline $50-60$ & Disc signal & 50 & 63 & $0.37(0.13$ to 0.61$)$ & $0.34(0.11$ to 0.57$)$ & & $0.36(0.18$ to 0.54$)$ \\
\hline$>60$ & Disc signal & 22 & 28 & $0.45(0.08$ to 0.81$)$ & $0.48(0.18$ to 0.78$)$ & & 0.41 (0.14 to 0.68$)$ \\
\hline$<50$ & Disc bulge & 18 & 53 & $0.39(0.0$ to 0.80$)$ & $0.10(0.00$ to 0.37$)$ & 0.28 (0 to 0.65$)$ & \\
\hline $50-60$ & Disc bulge & 50 & 63 & $0.56(0.38$ to 0.75$)$ & $0.16(0.00$ to 0.41$)$ & $0.51(0.28$ to 0.74$)$ & \\
\hline$>60$ & Disc bulge & 22 & 28 & $0.55(0.24$ to 0.86$)$ & $0.34(0.00$ to 0.68$)$ & $0.53(0.13$ to 0.94$)$ & \\
\hline$<50$ & Anterior osteophytes & 18 & 53 & $0.71(0.49$ to 0.94$)$ & $0.40(0.18$ to 0.63$)$ & $0.74(0.42$ to 1.0$)$ & \\
\hline $50-60$ & Anterior osteophytes & 50 & 63 & $0.58(0.40$ to 0.77$)$ & $0.58(0.42$ to 0.75$)$ & & $0.58(0.43$ to 0.74$)$ \\
\hline$>60$ & Anterior osteophytes & 22 & 28 & $0.60(0.31$ to 0.89$)$ & $0.56(0.29$ to 0.82$)$ & & $0.57(0.32$ to 0.81$)$ \\
\hline$<50$ & Summary score & 18 & 53 & 0.57 (0.24 to 0.89$)$ & $0.12(0.00$ to 0.39$)$ & 0.40 (0.06 to 0.73$)$ & \\
\hline $50-60$ & Summary score & 50 & 63 & $0.42(0.19$ to 0.65$)$ & $0.31(0.08$ to 0.55$)$ & $0.48(0.24$ to 0.72$)$ & \\
\hline$>60$ & Summary score & 22 & 28 & $0.39(0.00$ to 0.77$)$ & $0.48(0.18$ to 0.78$)$ & & $0.40(0.13$ to 0.67$)$ \\
\hline
\end{tabular}

Heritability was calculated using 90 monozygotic and 144 dizygotic same-sex twin pairs (omitted when dizygotic intraclass correlation greater than monozygotic intraclass correlation clearly suggests no heritable component) when $\mathrm{N}$ is the sample size in twin pairs by age subgroup. Age category $0=$ age $<50$ years, category $1=$ age. $\Delta$ Trait/time per year represents change in trait per year. A represents additive genetic, $\mathrm{C}$ common environment and $\mathrm{E}$ unique environmental factors.

significantly greater deterioration in LDD than monozygotic twins ( 10.55 vs $8.95, p=0.0002)$ and, adjusting for the time interval between the two MRI scans, a greater rate of progression (1.0 vs 0.84 per year, $\mathrm{p}<0.001)$. BMI, age at baseline and gender had no detectable effect on the progression of LDD over 10.7 years.

\section{Heritability of progression of LDD}

Table 3 shows the intraclass correlation coefficients for the monozygotic and dizygotic twins by MRI trait, together with the associated heritability estimates derived from modelling. Intraclass correlations being greater in monozygotic than dizygotic twins is indicative of a genetic influence on a trait. Disc height progression showed no evidence of influence by genetic factors in any age stratum. In the other traits there was evidence of genetic influence particularly in the younger age group: disc signal in those less than 50 years had a high heritability estimate of $76 \%$. In contrast, disc bulge was found to be heritable at all three age categories, with greater heritability in older subjects, range $28(0-0.65 \%)$ to $53(0.13-0.94 \%)$. The progression of osteophytes in those less than 50 years showed a marked genetic effect $(0.74(0.42-1.05))$ with no genetic influence detectable in the older age categories. Unsurprisingly, the heritability of the summary score progression reflects that of its components and is mainly influenced by genetic factors at younger age.

\section{DISCUSSION}

This is the first report of the heritability of progression of LDD and we have an extended period of follow-up of over 10 years in our sample of monozygotic and dizygotic twins. The main findings were that all the MRI-determined traits deteriorated over the decade and genetic influences on progression varied across the traits as well as by age. For example, change in disc height did not appear to be influenced by genetic factors at any age while disc bulge was heritable in all age categories. Deterioration of disc signal intensity and osteophytes had a significant genetic influence at young age only, which may be a reflection of the greater variation in progression in this age group. We previously reported no significant heritability for disc signal intensity based on the cross-sectional (baseline) MRI findings in this cohort. ${ }^{6}$ Taken together, these data suggest that any genetic effect on disc signal occurs early rather than late in adult life. This is not without precedent: heritability of a number of other traits, including musculoskeletal traits, is known to vary with age. For example, there is no detectable genetic contribution to hip fracture in older people (over 80 years), whereas the trait is clearly heritable in younger people, with estimates from a large twin study in Sweden suggesting heritability as high as $68 \% .^{10}$

The progression of LDD over a 5 -year time period has been investigated previously by plain radiography in UK singletons, ${ }^{5}$ which showed a $4-5 \%$ progression rate, and by MRI in 134 male monozygotic twins from Finland showing 3-4\% progression. ${ }^{11}$ The latter study highlighted different MRI-determined traits progressing at different rates. As the Finnish study did not include dizygotic twins, the heritability of progression could not be estimated but the investigators used the less specific measure of 'familial aggregation' determined from intraclass correlations. 

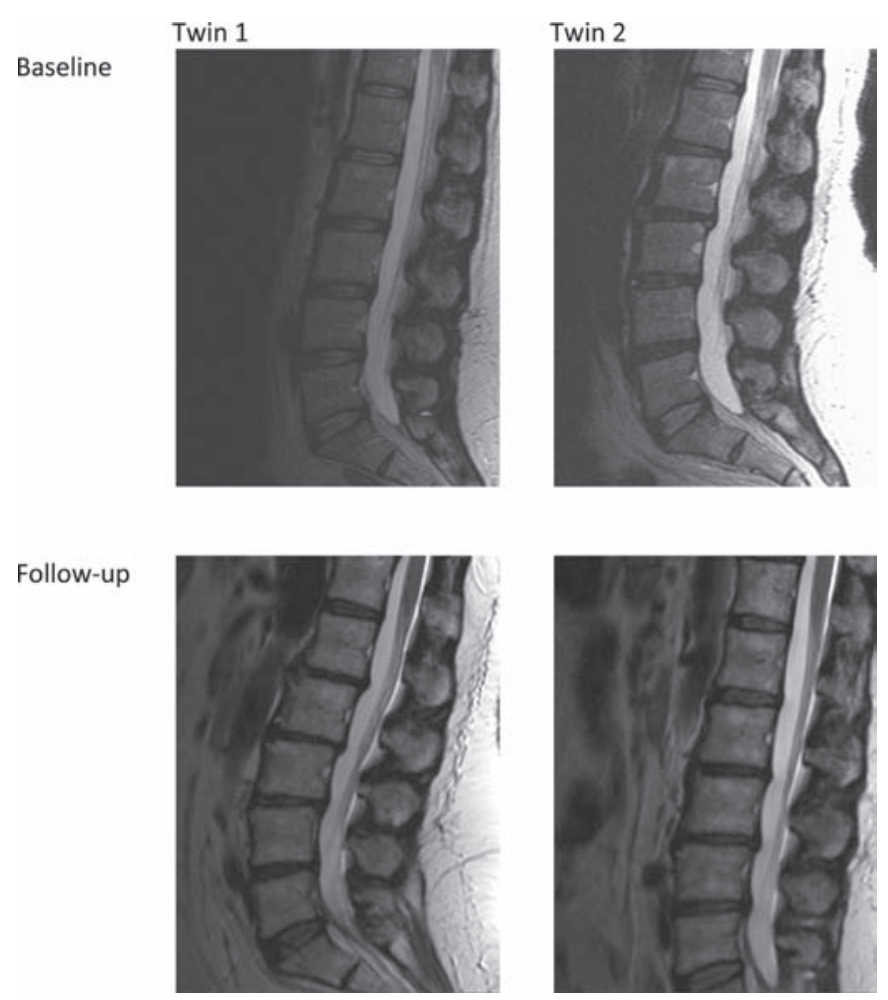

Figure 1 Illustrative MRI lumbar spine scans from a pair of monozygotic twins. Sagittal T2-weighted MRI scans from a pair of female monozygotic twins, baseline scan uppermost. Aged 52 years at baseline, both follow-up scans show increasing loss of disc signal intensity, loss of disc height and increasing extent and severity of disc bulges posteriorly.

Their findings of MRI-determined posterior disc bulge progression not being genetic are different to ours, as were their results for disc height. ${ }^{11}$ However, the Finnish study was of a considerably smaller sample ( $n=67$ twin pairs; 3.5 times smaller than the sample reported here) over a shorter time period (5 years) and the authors did not study changes in signal intensity. The other major difference between the two cohorts is in the gender of the participants, so perhaps the contrasting findings reveal a true sex difference in LDD progression. Our findings highlight the heterogeneity of the MRI traits, and suggest that they reflect very different features of the pathological process and are under differential control. This suggests that future studies should continue to examine these features separately. This is in keeping with findings from the osteoarthritis literature-the approach is commonly referred to as a 'splitting' rather than 'lumping' method. These terms have also been used to describe the practice of summing osteoarthritis across body sites rather than considering each individually. Support for this approach is mounting, with the accumulating evidence of differential genetic influences at different anatomical sites. ${ }^{12} 13$

We acknowledge a number of limitations to this study. As our sample was predominantly of female subjects it does not, on the whole, contain subjects reporting heavy manual occupations, although such environmental factors are now considered to play a small role. LDD is thought to be similar across the sexes; although site and sex-specific genetic predisposition is being identified for peripheral joint osteoarthritis, there is little evidence of a significant structural difference between the sexes in LDD. Men are thought to have earlier degenerative change based on a postmortem study from the 1920s, ${ }^{14}$ but as we detected no significant difference in LDD between the men and the women in our sample, we felt it reasonable to include both groups in our study. These twins have been shown to be representative of the UK population for a range of traits including degenerative joint diseases. ${ }^{15}$ Twins were not recruited on the basis of back pain but the possibility that this may have been a motivation to participate cannot be fully discounted. A concern in these data was that there was a relatively poor measure of intercoder reproducibility $(\kappa=0.54)$ and systematic differences in the monozygotic and dizygotic groups. The latter is partly the result of slightly younger dizygotic twin volunteers, and while this is probably a chance finding, it is possible that a degree of selection bias introduced systematic differences. The intercoder $\kappa$ is not likely to have influenced findings as almost all the baseline scans were coded by PNS and the follow-up scans by MP. Systematic bias in the scans and their coding could have influenced the rate of progression of LDD-newer MRI scans were inevitably of higher quality than the baseline scan-but one would not expect a differential between monozygotic and dizygotic twins, so heritability would not be affected.

Our initial data analysis showed that the effect of age on the rate of degeneration was non-linear. Furthermore, there was a suggestion of a ceiling effect in the coding for degenerative change, with more severe disease progressing less rapidly. As a result, we felt that it was most appropriate to examine heritability in age strata, rather than by attempting to eliminate age effects using regression modelling. This approach necessarily reduced the statistical power, and is reflected in the wide CI of the stratum-specific heritability estimates (table 3 ). While definitive statements regarding the genetic influence on the progression of MRI traits at differing age groups should be made with caution, the results across traits are suggestive of a change in genetic influence over age.

That genetic factors underlie the rate of progression of LDD is not, perhaps, surprising in itself, but is of importance because it provides the rationale to the search for precise genetic variants controlling LDD progression. In addition, progression of LDD may be more closely related to symptoms of low back pain than cross-sectional degeneration-there is evidence that this is the case in similar phenotypes such as knee osteoarthritis. ${ }^{16}$ These results will direct the search for genetic variants influencing the rate of deterioration as, arguably, those at risk of rapid progression are the most important group to identify when interventions become available for trials. Cross-sectional LDD is highly heritable and has been the subject of a number of candidate gene studies and variants identified that have been replicated in one or more studies include VDR, COL9A2, COL9A3, COL1A1, MATN3, MMP and some inflammatory genes such as IL-1 and THSD2. ${ }^{17}$ However, in common with many complex traits, the genetic variants described thus far account for only a small proportion of the estimated heritability, and it is likely that many will turn out to be false positives, as has been reported for osteoporosis. $^{18}$

Little is known of the environmental factors governing the rate of progression of LDD. BMI is strongly suspected if not proved, smoking and occupational maximum lifting have been shown to influence progression in twin studies. ${ }^{19}$ Good longitudinal epidemiological studies remain scarce (for cross-sectional review see Williams and Sambrook). ${ }^{20}$ This information will be important information to obtain, for if the main effects are environmental then these may be modifiable by, for example, weight loss or occupational changes. If, however, most of the variation in progression is attributable to genetic factors, then further understanding of gene products may provide novel targets for intervention. In addition, the identification of new 
biological pathways may aid in the identification of much needed biomarkers for future trials of disease-modifying drugs. Low back pain is a considerable public health issue: new pathways to target would be of great interest to the pharmaceutical industry and to the many practitioners with a role in managing back pain. Our results point the way to subject selection in studies to identify genetic variants: they highlight that such changes are largely seen in young-middle age. Genetic variants influencing progression are now a realistic target for researchers into LDD. This study provides the impetus for further work to identify the genetic variants involved as well as a clinical assessment of whether those with rapid LDD progression are at greater risk of symptoms.

Acknowledgements The authors are indebted to the twin volunteers in the UK and Australia and also the Departments of Radiology at St Thomas' Hospital and the London Bridge Hospital for performing the MRI scans.

Funding This project was made possible by funding provided by Arthritis Research UK, the Wellcome Trust and the Medical Research Council.

Competing interests None.

Patient consent Obtained.

Ethics approval This study was conducted with the approval of the Guy's and St Thomas' Trust, King's College London, Royal North Shore Hospital.

Provenance and peer review Not commissioned; externally peer reviewed.

\section{REFERENCES}

1. Endean A, Palmer KT, Coggon D. Potential of magnetic resonance imaging findings to refine case definition for mechanical low back pain in epidemiological studies: a systematic review. Spine 2011;36:160-9.

2. MacGregor AJ, Andrew T, Sambrook PN, et al. Structural, psychological, and genetic influences on low back and neck pain: a study of adult female twins. Arthritis Rheum 2004;51:160-7.

3. Borenstein DG, O'Mara JW Jr, Boden SD, et al. The value of magnetic resonance imaging of the lumbar spine to predict low-back pain in asymptomatic subjects: a seven-year follow-up study. J Bone Joint Surg Am 2001;83-A:1306-11.
4. Jensen MC, Brant-Zawadzki MN, Obuchowski N, et al. Magnetic resonance imaging of the lumbar spine in people without back pain. N Engl J Med 1994;331:69-73.

5. Hassett G, Hart DJ, Manek NJ, et al. Risk factors for progression of lumbar spine disc degeneration: the Chingford Study. Arthritis Rheum 2003;48:3112-17.

6. Sambrook PN, MacGregor AJ, Spector TD. Genetic influences on cervical and lumbar disc degeneration: a magnetic resonance imaging study in twins. Arthritis Rheum 1999;42:366-72.

7. Spector TD, Williams FM. The UK Adult Twin Registry (TwinsUK). Twin Res Hum Genet 2006;9:899-906.

8. Makovey J, Nguyen TV, Naganathan V, et al. Genetic effects on bone loss in peri- and postmenopausal women: a longitudinal twin study. J Bone Miner Res 2007;22:1773-80.

9. Jarosz JM, Bingham JB, Pemberton J, et al. An atlas for scoring cervical and lumbar disc degeneration. London: Springer-Verlag, 1997.

10. Michaëlsson K, Melhus $\mathrm{H}$, Ferm H, et al. Genetic liability to fractures in the elderly. Arch Intern Med 2005;165:1825-30.

11. Videman T, Battié MC, Parent $\mathrm{E}$, et al. Progression and determinants of quantitative magnetic resonance imaging measures of lumbar disc degeneration: a five-year follow-up of adult male monozygotic twins. Spine 2008;33:1484-90.

12. MacGregor AJ, Li Q, Spector TD, et al. The genetic influence on radiographic osteoarthritis is site specific at the hand, hip and knee. Rheumatology (Oxford) 2009; 48:277-80.

13. Valdes AM, McWilliams D, Arden NK, et al. Involvement of different risk factors in clinically severe large joint osteoarthritis according to the presence of hand interphalangeal nodes. Arthritis Rheum 2010;62:2688-95.

14. Battié MC, Videman T. Lumbar disc degeneration: epidemiology and genetics. J Bone Joint Surg Am 2006;88(Suppl 2):3-9.

15. Andrew T, Hart DJ, Snieder H, et al. Are twins and singletons comparable? A study of disease-related and lifestyle characteristics in adult women. Twin Res 2001:4:464-77.

16. Hunter DJ, Zhang W, Conaghan PG, et al. Systematic review of the concurrent and predictive validity of MRI biomarkers in OA. Osteoarthritis Cartilage 2011; In press.

17. Ryder JJ, Garrison K, Song F, et al. Genetic associations in peripheral joint osteoarthritis and spinal degenerative disease: a systematic review. Ann Rheum Dis 2008;67:584-91.

18. Richards JB, Kavvoura FK, Rivadeneira F, et al. Collaborative meta-analysis: associations of 150 candidate genes with osteoporosis and osteoporotic fracture. Ann Intern Med 2009;151:528-37.

19. Battié MC, Videman T, Kaprio J, et al. The Twin Spine Study: contributions to a changing view of disc degeneration. Spine J 2009;9:47-59.

20. Williams FMK, Sambrook PN. Neck and back pain and intervertebral disc degeneration: role of occupational factors. Best Practice \& Research Clinical Rheumatology 2011; doi:10.1016/j.berh.2011.01.007. 